\title{
US survey sets cosmic priorities
}

\section{Dark energy rises to the top in decadal report ranking future astronomy and astrophysics projects.}

Recently, a colleague of astronomer Claire Max jokingly told her that, come 13 August, half her friends would love her and half would never want to speak to her again.

That is because Max, of the University of California, Santa Cruz, has for the past two years been helping to craft US astronomy's latest decadal survey, an influential report prepared for the National Research Council that recommends which astronomy and astrophysics projects NASA, the National Science Foundation (NSF) and the Department of Energy (DOE) should fund over the next ten years.

Using input from 9 appointed panels, 17 town-hall meetings and 324 white papers from individual research groups, the survey's aim is to assess opportunities and set priorities for US astronomy and astrophysics, while balancing scientific goals with fiscal realities. The report is now out, and although Max isn't aware of losing any friends, there are some in the community who have more reason to thank her than others.

Topping the list of the pleased and grateful is Anthony Tyson, an astrophysicist at the University of California, Davis, and director of the proposed Large Synoptic Survey Telescope (LSST), which ranks highest among the ground-based facilities considered by the survey committee. When completed in 2015, the 8.4-metre telescope will regularly sweep the entire visible sky in three nights with a 3.2-gigapixel camera, capturing short-lived phenomena ranging from fast-moving near-Earth asteroids to the flashes of supernovae in distant galaxies.

Over ten years, the US $\$ 465$ million observatory will also build up an unprecedented 100 -petabyte database for astronomers trying to discern the nature of two mysterious factors that shape the Universe. One is dark matter, thought to be an unknown particle or family of particles beyond the standard model of physics. Hidden in vast quantities among the galaxies, dark matter generates a gravitational pull that has shaped the evolution of the Universe. The other factor is dark energy, the pervasive but mysterious phenomenon that is causing cosmic expansion to accelerate. Crucial data on both factors can be derived from a three-dimensional survey of the surrounding Universe that the LSST is well suited to provide.

"Increasingly, we are able to ask new questions by querying huge databases," says Tyson. "The key is to populate those databases with calibrated and trusted data."

The LSST is expected to help US astronomers regain some momentum in ground-based

Such data would contain subtle clues - in the distance-brightness relationships of supernovae, the bending of light (microlensing) from background galaxies and the three-dimensional clustering of matter in space - that can be used to independently measure dark energy.

WFIRST is effectively a rebranding of the Joint Dark Energy Mission, a NASA-DOE collaboration. The new name, says one survey reviewer, signals that the $\$ 1.6$-billion telescope is not a one-trick pony, but a way of serving other astronomical needs as well. The survey committee stresses, for example, that WFIRST could spot microlensing events caused when exoplanets - planets outside our Solar System - pass briefly in front of background stars in the Milky Way. Although the method is unsuitable for studying individual solar systems in detail, it promises, through its sheer number of discoveries, to provide an unbiased sample of the kinds of planetary systems prevalent in the Galaxy.

This may not be enough to satisfy those who study exoplanets. "I don't think the report reflects that activity and the amount of young

astronomy at a time when European facilities have begun to dominate the field. To that end, the survey stresses the need for a swift decision on which of two competing mega-telescopes should receive federal funding.

The proposed Thirty Meter Telescope, on Mauna Kea in Hawaii, and the Giant Magellan Telescope, envisioned for Las Campanas in Chile, are both supported by significant private money, and would have many times the light-gathering power and resolution of today's largest telescopes. Realistically, only one project will receive federal funds, which the survey recommends should be between $\$ 257$ million and $\$ 350$ million. Given that Europe has also prioritized a 42 -metre telescope, the European Extremely Large Telescope, a choice needs to be made now to avoid a counterproductive stalemate.

In space, the decadal survey proposes the Wide Field Infrared Survey Telescope (WFIRST), a 1.5-metre instrument that will map the whole sky at near-infrared wavelengths. people going into exoplanets," says Sara Seager, an astrophysicist at the Massachusetts Institute of Technology in Cambridge. Seager notes that the survey abandons support for the Space Interferometry Mission, a project that would have detected planets slightly larger than Earth through their gravitational effect on the stars they orbit. However, Seager applauds another recommendation: a \$100-million to \$200-million allocation for the technical development of a future exoplanet mission.

Exoplanet researchers were stung when the Terrestrial Planet Finder, a mission endorsed by the previous decadal survey was later cancelled owing to cost overruns. So they welcome the chance to continue pursuing other approaches to observing Earth-like planets.

"Last week, I was worried," says Webster Cash, who is working on an exoplanet-mission concept at the University of Colorado, Boulder. "Now, I’m feeling like my career is going to be a lot of fun for the next ten years."

Adam Mann

See Editorial, page 903. For a full list of ranked projects, see go.nature.com/BrYViu. 